\title{
Carboxymethyl chitosan-graft-phosphatidylethanolamine: Amphiphilic matrices for controlled drug delivery
}

\author{
M. Prabaharan, R.L. Reis, J.F. Mano * \\ 3B's Research Group-Biomaterials, Biodegradables and Biomimetics, University of Minho, Campus of Gualtar, 4710-057 Braga, Portugal \\ Department of Polymer Engineering, University of Minho, Campus of Azurém, 4800-058 Guimarães, Portugal
}

Received 10 October 2005; received in revised form 7 September 2006; accepted 14 September 2006 Available online 19 October 2006

\begin{abstract}
Modified carboxymethyl chitosan (CMC) containing phosphatidylethanolamine (PEA) groups were synthesized by a 1ethyl-3-(3-dimethylaminopropyl)carbodiimide (EDC)-mediated coupling reaction. The structure of the modified CMC exhibiting an amphiphilic character was analysed by FT-IR and ${ }^{1} \mathrm{H}$ NMR. CMC-g-PEA beads were prepared with sodium tripolyphosphate (TPP) by ionic-crosslinking. The beads sizes were in range from 800 to $1200 \mu \mathrm{m}$ and encapsulation efficiencies of drug were more than $68 \%$. The morphologies of CMC-g-PEA beads were examined with scanning electron microscopy (SEM). The release experiments were performed using ketoprofen as an hydrophobic model drug. The drug dissolution kinetics showed longer release times for CMC-g-PEA beads: $20 \mathrm{~h}$ (at pH 1.4) and $45 \mathrm{~h}$ (at pH 7.4). The amount of the drug release was much higher in acidic solution than in basic solution due to the swelling properties of the matrix at acidic $\mathrm{pH}$. These results suggest that modified CMC with PEA may become a potential delivery system to control the release of hydrophobic drugs.
\end{abstract}

(C) 2006 Elsevier B.V. All rights reserved.

Keywords: Carboxymethyl chitosan; Phosphatidylethanolamine; Amphiphilic; Drug delivery

\section{Introduction}

Interest in polymeric matrices for pharmaceutical formulation continues to grow specially those that react to external stimuli (e.g. $\mathrm{pH}$ or temperature), allowing to target the release of drugs in specific conditions or site. Special attention is currently given to chitosan, a poly(aminosaccharide), normally obtained by partial alkaline deacetylation of

\footnotetext{
* Corresponding author. Tel.: +351 253510330; fax: +351 253510339.

E-mail address: jmano@dep.uminho.pt (J.F. Mano).
}

chitin, the principle component of living organisms such as fungi and crustacean. This naturally occurring polymer has a repeating structural unit of 2acetamido-2-deoxy- $\beta$-D-glucose [1,2]. Since chitosan itself is non-toxic [3], biodegradable [4] and biocompatible [5], several biological applications have been reported for chitosan, including chelation processes [6], a cholesterol trap [7] and a drug carrier [8,9]. However, chitosan is only soluble in a few dilute acid solutions, which limits its wide applications. Recently, there has been a growing interest in chemical modification of chitosan to improve its solubility and widen its applications $[10,11]$. The chemical 
modification is a powerful tool to control the interaction of the polymer with drugs, to enhance the drug loading efficiency as well as tailor the drug release period. Chemically modified chitosans have great utility in controlled release and targeting studies of almost all class of bioactive molecules. Therefore, the interest in chitosan and its derivatives as excipients in drug delivery has been increased in recent years. Chitosan has been modified by ioniccrosslinking (e.g. with tripolyphosphate, TPP) to prepare intelligent drug delivery systems [12], and by carboxymethylation for repair and regeneration of bone tissues [13] or use as antioxidant agent [14]. Chitosan has also been conjugated with $\alpha$ galactosyl for use as an inhibitor in acute rejection following xenotransplantation [15], with vinylsulfonate as an antimicrobial agent [16], and with sulfate as an activator of blood anticoagulant factors [17].

Self-assembled nano/microparticles, composed of polymeric amphiphiles, have been considered to provide opportunities for the site-specific delivery of drugs because they can solubilize various hydrophobic drugs, increase bioavailability, and stay unrecognized during blood circulation $[18,19]$. In this context, chitosan was acylated with fatty acyl chlorides to introduce hydrophobicity for use as a matrix for drug delivery and glycol chitosan was hydrophobically modified with deoxycholic acid, $5 \beta$-cholanic acid and doxorubicin to yield selfaggregates as potential drug carriers [20-23]. Glycol chitosan was also modified with palmitoyl $\mathrm{N}$ hydroxysuccinimide to form vesicles [24] and hydrogels [25]. These derivatives present amphiphilic characteristics, stabilized by hydrophobic interactions, and exhibit erosion-controlled drug release for 5-7 h. Recently, we have synthesized a $\mathrm{pH}$ responsive chitosan derivative bearing $\beta$-cyclodextrin cavities to be used as a matrix for controlled drug release [26]. Due to the presence of hydrophobic $\beta$-cyclodextrin rings, this system provided a slower release of the entrapped hydrophobic drug. In the present study, water soluble carboxymethyl chitosan (CMC) was prepared from chitosan and hydrophobically modified by covalent attachment of phosphatidylethanolamine (PEA) to CMC through amide bond formation. This system may form polymeric micelles composed by hydrophobic PEA segments as the internal core and hydrophilic CMC segments as a surrounding corona in an aqueous solution. It was expected that such derivatization would reduce hydration of the matrix and play a role in network stabilization by hydrophobic interactions. For the drug delivery study, CMC- $g$ PEA beads were prepared based on ionic gelation method by using sodium tripolyphosphate (TPP) as an ionic-crosslinking agent [27]. The feasibility of these beads as a delivery carrier for the transfection of hydrophobic model drug, ketoprofen was investigated. The morphological observations and swelling properties of beads were evaluated. We believe that the main conclusions that may be obtained using beads could be extended to other geometrical models, such as nano/microparticles, bulk hydrogels or porous three-dimensional constructs in order to be used in tissue engineering applications.

\section{Materials and method}

\subsection{Materials}

Chitosan (viscosity average molecular weight $6.7 \times 10^{5} \mathrm{~g} / \mathrm{mol}$, degree of $N$-deacetylation $75-85 \%$ ) was purchased from Sigma Chemical Company. Before used, it was purified by the method of dissolving in acetic acid and separating with alkali. PEA and EDC were from Sigma-Aldrich and used without further purification. Chloroacetic acid, TPP and sodium hydroxide were supplied by Fluka Chemical Company and used as supplied. All other chemicals used were of analytical reagent grade.

\subsection{Preparation of $C M C$}

CMC was prepared according to a method described by Chen et al. [28]. Purified chitosan $(8.0 \mathrm{~g})$ was added into $40 \mathrm{~g}$ of $50 \mathrm{wt} \% \mathrm{NaOH}$ solution and put into a refrigerator at $-18{ }^{\circ} \mathrm{C}$ overnight for alkylization. After the excessive alkali solution was extruded, chitosan was put into a $250 \mathrm{ml}$ reactor containing $40 \mathrm{ml}$ of isopropyl alcohol. Then, $10 \mathrm{~g}$ of chloroacetic acid dissolved in $40 \mathrm{ml}$ of isopropyl alcohol was added into the reactor drop wise. Afterwards, this reaction mixture was refluxed at $65{ }^{\circ} \mathrm{C}$ under extensive stirring. After an additional $2 \mathrm{~h}$ the reaction was stopped and the solvent was discarded. The mixture was neutralized with hydrochloric acid and then precipitated in acetone. The product was carefully washed successively by acetone, absolute alcohol and 75\% (v/v) alcohol and then dried at $60{ }^{\circ} \mathrm{C}$ under vacuum. The elemental analysis showed that the substitution degree of carboxymethylation of $\mathrm{CMC}$ is 0.65 . Analytical and calculated data for $\left[\mathrm{C}_{6} \mathrm{H}_{8} \mathrm{O}_{4}\left(\mathrm{NH}_{2}\right)_{0.85}\right.$ (NHC- 
$\left.\left.\mathrm{OCH}_{3}\right)_{0.15}\left(\mathrm{C}_{2} \mathrm{H}_{3} \mathrm{O}_{2}\right)_{0.65}(\mathrm{H})_{0.35} \cdot 1.6 \mathrm{H}_{2} \mathrm{O}\right]_{n}$ was determined as $\mathrm{C} 39.10, \mathrm{~N} 6.04, \mathrm{H} 6.72$ and $\mathrm{C} 39.04, \mathrm{~N}$ 5.99, H 6.67, respectively.

\subsection{Preparation of $C M C-g-P E A$}

PEA (300 mg) in the mixture of toluene and methanol (5:1 ratio) was added to a $1 \mathrm{wt} \%$ carboxymethyl chitosan solution followed by the drop wise addition of EDC $(0.71 \mathrm{~g})$ under constant stirring at room temperature. After 15 hours, the reaction mixture was poured into the acetone. The precipitates were filtered off, washed thoroughly with methanol and acetone, and then followed by vacuum drying at room temperature. Elemental analysis showed a significant reduction of $\mathrm{N}$ content when comparing the obtained product $(3.71 \%)$ with pure CMC $(6.04 \%)$. The $\mathrm{N}$ content of the product corresponds to a degree of substitution $62 \%$, showing that most of the carboxyl groups in CMC were grafted with amine groups of PEA. The degree of substitution was calculated from the $\mathrm{N}$ content of the product with the following equation:

$[14-(217 \times \mathrm{N} \%)] /[(635 \times \mathrm{N} \%)-14]$

where 217 is the weight of one gluco-pyranoside moiety of $\mathrm{CMC}$ molecule after deducting $\mathrm{OH}^{-}$from carboxymethyl group, 14 is the weight of $\mathrm{N}$ in the CMC and PEA, and 635 is the molecular weight of PEA after deducting $\mathrm{H}$ atom.

\subsection{Characterization}

The IR spectra of CMC and its derivatives were recorded with a double-beam Perkin-Elmer 1600 FT-IR spectrometer in the range of 4000$400 \mathrm{~cm}^{-1}$ using $\mathrm{KBr}$ pellets. ${ }^{1} \mathrm{H}$ NMR spectrum of the samples was recorded on a Varian Unity Plus $300 \mathrm{MHz}$ spectrometer in $\mathrm{D}_{2} \mathrm{O}$ at $25^{\circ} \mathrm{C}$. The samples were dissolved in a $5 \mathrm{~mm}$ diameter tube at a concentration of $20 \mathrm{mg} / \mathrm{ml}$. For the control PEA, $\mathrm{CDCl}_{3}$ was used as a solvent. The elemental analyses were carried out with a Leco CHNS 932 instrument. The morphology of CMC-g-PEA beads was analysed using a Leica Cambridge S360 SEM at an accelerating voltage of $15 \mathrm{kV}$. Absorbance measurements were carried out in a Shimadzu UV-visible spectrophotometer model UV-1601. A calibration curve of absorbance against different concentrations of ketoprofen was made at $267 \mathrm{~nm}$.
2.5. Preparation of drug loaded crosslinked CMC-gPEA beads

CMC- $g$-PEA solution was prepared by dissolving $1.0 \mathrm{~g}$ of CMC-g-PEA in $100 \mathrm{ml}$ of distilled water. With this solution, a known amount of ketoprofen $(0.5$ and $1 \mathrm{~g})$ in $5 \mathrm{ml}$ of ethanol was added and dispersed under constant stirring. This mixture was dropped through a syringe into $100 \mathrm{ml}$ of $10 \%$ (w/v) TPP aqueous solution and the gelled spheres formed instantaneously. During this process, $\mathrm{pH}$ of the TPP solution was maintained at 4.0 by using dilute $\mathrm{HCl}$. The formed CMC-g-PEA beads remained in this solution for $1 \mathrm{~h}$ and were then washed with deionized water repeatedly, and finally dried at room temperature. A similar method is followed for the preparation of control chitosan beads loaded with drug.

\subsection{Determination of initial drug content}

A weighed quantity $(50 \mathrm{mg}$ ) of drug loaded beads was extracted with ethanol at $37^{\circ} \mathrm{C}$ for $48 \mathrm{~h}$ under uniform stirring. After centrifugation, the ketoprofen in the supernatant was assayed by UV-visible spectrophotometer at a wavelength of $267 \mathrm{~nm}$. All the experiments were carried out in triplicate.

\subsection{Swelling studies}

The swelling behaviour of the chitosan and CMC- $g$-PEA beads was studied in solutions of different $\mathrm{pH}$ at $37^{\circ} \mathrm{C}$. The beads were accurately weighed $(50 \mathrm{mg})\left(W_{0}\right)$ and immersed in solutions of $\mathrm{pH} 1.4$ and 7.4. At predetermined time intervals the swollen beads were weighed after they were wiped with soft paper tissue $\left(W_{t}\right)$. The degree of swelling for each sample at time $t$ was calculated by using the expression: $\left(W_{t}-W_{0}\right) / W_{0} \times 100$, where $W_{t}$ and $W_{0}$ are the weights of the beads at time $t$ and in the dry state, respectively.

\subsection{Drug release studies}

The release studies were performed in a glass apparatus at $37^{\circ} \mathrm{C}$ in $\mathrm{HCl}-\mathrm{KCl}(\mathrm{pH} \mathrm{1.4)}$ and phosphate buffer ( $\mathrm{pH}$ 7.4) solutions. Drug loaded beads $(15 \mathrm{mg}$ ) were suspended in $100 \mathrm{ml}$ of release medium and stirred at $100 \mathrm{rpm}$ in a horizontal laboratory shaker and maintained at $37^{\circ} \mathrm{C}$ in a water bath. Samples $(2 \mathrm{ml})$ were periodically removed, filtered and assayed. The volume of each sample withdrawn 
was replaced by the same volume of fresh medium. The amount of released ketoprofen was analyzed with a spectrophotometer at $267 \mathrm{~nm}$. The drug release studies were performed in triplicate for each of the samples.

\section{Results and discussion}

The water soluble carbodiimide, EDC, is a socalled 'zero-length' crosslinker because the amide linkages are formed without leaving a spacer molecule. EDC reacts with carboxyl groups of CMC to form an active ester intermediate. The intermediate can react with a primary amine of PEA to form an amide bond. Isourea is a by-product and can be easily removed by washing with water. The reaction scheme for the preparation of CMC- $g$-PEA is given in Fig. 1.

\subsection{FT-IR and ${ }^{1} H$ NMR analysis}

The FT-IR spectrum of chitosan (Fig. 2a) shows a broad $-\mathrm{OH}$ stretch absorption band between 3450 and $3100 \mathrm{~cm}^{-1}$ and the aliphatic $\mathrm{C}-\mathrm{H}$ stretch between 2990 and $2850 \mathrm{~cm}^{-1}$. As the $-\mathrm{OH}$ stretch band and the aliphatic $\mathrm{C}-\mathrm{H}$ stretch band are aligned, it appears as a broad band from 3450 and $2850 \mathrm{~cm}^{-1}$ in the spectrum. Other major absorption band between 1220 and $1020 \mathrm{~cm}^{-1}$ represents the

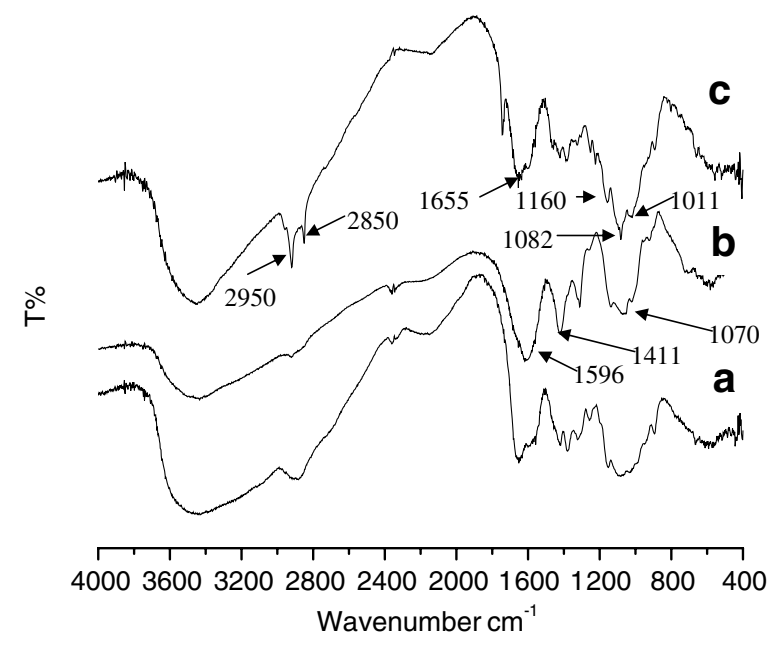

Fig. 2. FT-IR spectrum of (a) chitosan, (b) CMC and (c) CMC$g$-PEA.

free primary amino group $\left(-\mathrm{NH}_{2}\right)$ at $\mathrm{C}_{2}$ position. The peak at $1647 \mathrm{~cm}^{-1}$ represents acetylated amino group of chitin, which indicates that the sample is not fully deacetylated. The peak at $1384 \mathrm{~cm}^{-1}$ represents the $-\mathrm{C}-\mathrm{O}$ stretch of primary alcoholic group $\left(-\mathrm{CH}_{2}-\mathrm{OH}\right)$. In the IR spectrum of $\mathrm{CMC}$ (Fig. 2b), the strong peaks at 1596 and $1411 \mathrm{~cm}^{-1}$ could be assigned to the respective asymmetry and symmetry stretch vibration of $\mathrm{COO}^{-}$. Also, the $\mathrm{C}_{-}$ $\mathrm{O}$ adsorption peak of the secondary hydroxyl group

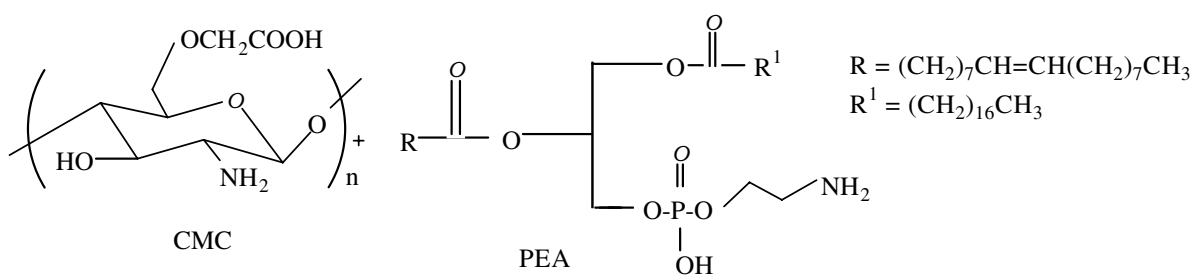

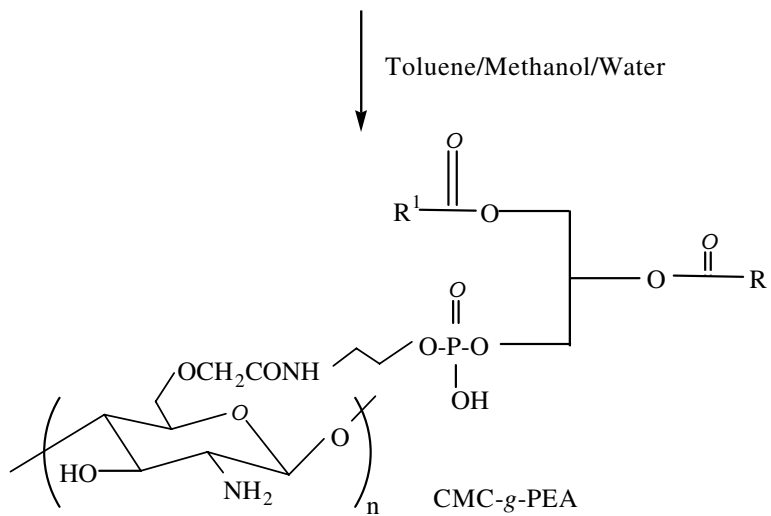

Fig. 1. Reaction scheme for the preparation of CMC- $g$-PEA. 
becomes stronger and moves to $1070 \mathrm{~cm}^{-1}$. The results indicate that the carboxymethyl substitution occurs at the $\mathrm{C}_{6}$ position of chitosan. In the IR spectrum of CMC-g-PEA (Fig. 2c), the absorption peak at $1655 \mathrm{~cm}^{-1}$ can be assigned to the carbonyl stretch of secondary amides (amide I band), at $1555 \mathrm{~cm}^{-1}$ to the $\mathrm{N}-\mathrm{H}$ bending vibration of the amide II band. In addition, peaks at 2850 and $2950 \mathrm{~cm}^{-1}$ were ascribed to the aliphatic chain $\left(-\mathrm{CH}_{2}\right)$. The absorption peak at $1730 \mathrm{~cm}^{-1}$ can be represented the $\mathrm{C}=\mathrm{O}$ stretch vibrations of carbonyl groups. Another absorption peaks at 1160,1082 and $1011 \mathrm{~cm}^{-1}$ can be represented the stretch vibrations of $\mathrm{P}=\mathrm{O}, \mathrm{P}-$ $\mathrm{OH}$ and $\mathrm{P}-\mathrm{O}$ of phosphate group respectively. Fig. 3 shows the ${ }^{1} \mathrm{H}$ NMR spectra of CMC, PEA and CMC-g-PEA. As shown in the spectrum of
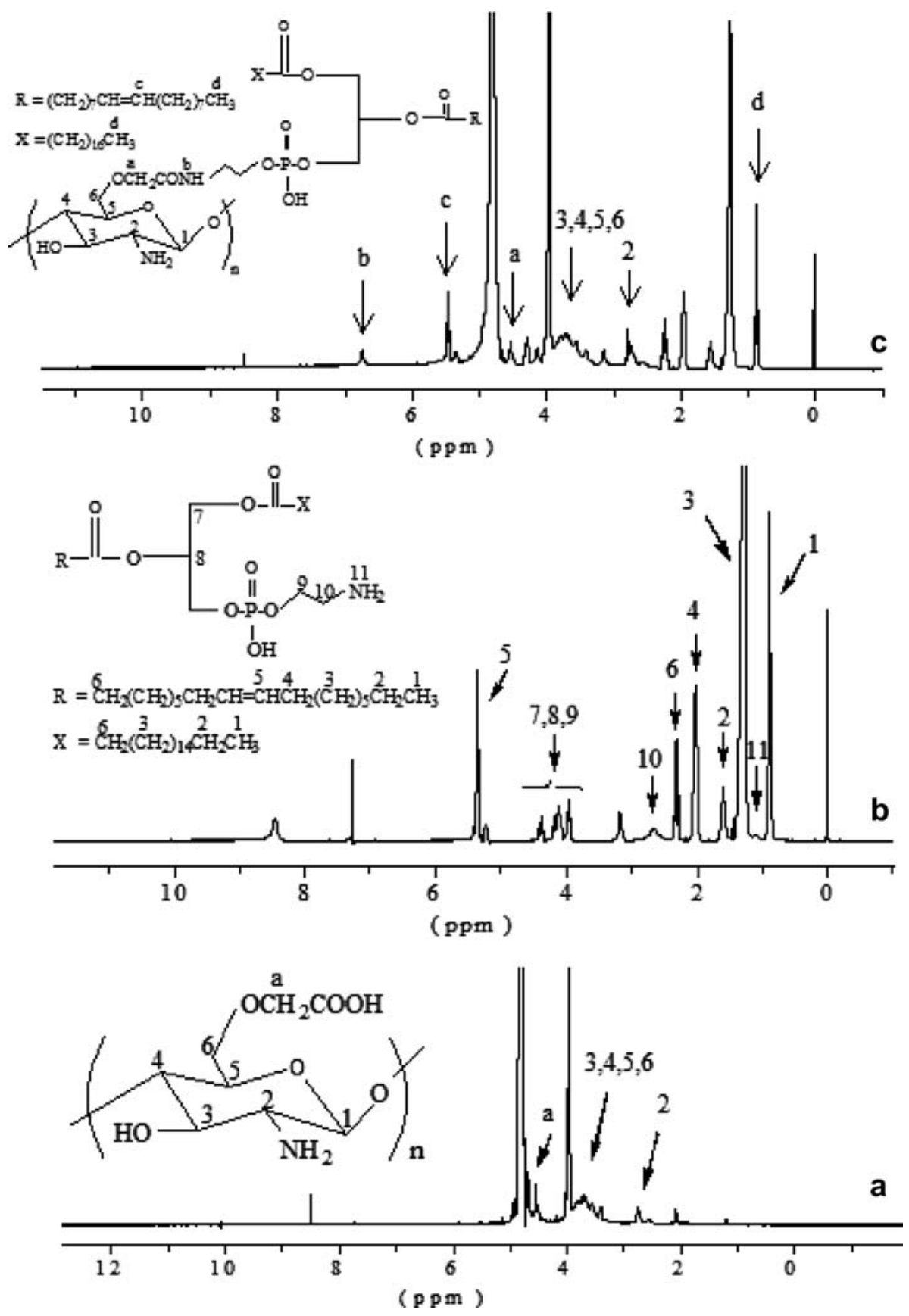

Fig. 3. ${ }^{1} \mathrm{H}$ NMR spectrum of (a) CMC, (b) PEA and (c) CMC-g-PEA. 
CMC (Fig. 3a), the chemical shifts at $4.5 \mathrm{ppm}$ was the protons of $-\mathrm{CH}_{2}-\mathrm{COO}^{-}$at the $\mathrm{C}_{6}$ position of the chitosan derivative (CMC). This indicated that carboxymethyl substitute was observed on some of the primary hydroxyl sites of the modified chitosan structure. The spectrum of PEA (Fig. 3b) exhibited peaks at $0.9 \mathrm{ppm}$ due to the presence of methyl groups, $1.24 \mathrm{ppm}$ due to the presence of amine group, $1.26 \mathrm{ppm}$ due to the methylene groups, and 5.2-5.4 ppm due to the vinyl proton. The signal around at $7.2 \mathrm{ppm}$ is ascribed to $\mathrm{CDCl}_{3}$. Fig. $3 \mathrm{c}$ shows the spectra of CMC- $g$-PEA copolymers. The signals at $3.2-4.2 \mathrm{ppm}$ are multiplet and those are originated from CMC. The signals due to $\mathrm{CH}_{3}$, methylene and vinyl protons of PEA also appeared in the spectrum of CMC- $g$-PEA copolymer. Also, a new peak at $6.7 \mathrm{ppm}$ was observed as the amide group $(-\mathrm{CONH}-)$ was formed in the CMC-g-PEA. These results clearly confirmed that PEA has been successfully graft copolymerized into CMC.

\subsection{Properties of CMC-g-PEA beads}

According to previous studies, drug release from chitosan particles could be controlled by crosslinking the matrix using chemical crosslinking agents such as glutaraldehyde or ethylene glycol diglycidyl ether $[29,30]$. However, these chemical crosslinking agents have possibility of inducing undesirable effects due its toxicity [31]. To overcome this disadvantage of chemical crosslinking, ionic-crosslinking interaction has been proposed [32,33]. It is reported that the beads prepared with TPP increased the drug loading efficiency as well as prolonging the drug release period [33]. TPP is non-toxic and multivalent anion. Therefore, in this study, CMC- $g$ PEA beads were prepared by the ionic interaction between a positively charged amino group of CMC- $g$-PEA and a negatively charged counter ion of TPP. The interaction could be controlled by the charge density of TPP and CMC-g-PEA, which is dependent on the $\mathrm{pH}$ value of solution [34]. In original TPP solution ( $\mathrm{pH}$ 8.6), TPP is dissociated into $\mathrm{OH}^{-}$and TPP ions $\left(\mathrm{HP}_{3} \mathrm{O}_{10}^{4-}\right.$ and $\left.\mathrm{P}_{3} \mathrm{O}_{10}^{5-}\right)$. However in low pH, only $\mathrm{P}_{3} \mathrm{O}_{10}^{5-}$ anions are present. Moreover, CMC-g-PEA is a weak polybase, and as $\mathrm{pH}$ of the solution decreases, the ionization of amine group of CMC-g-PEA increases. Therefore, one would expect that CMC- $g$-PEA beads prepared in the original TPP solution can be dominated by deprotonation and slightly ionic-crosslinking, but
CMC- $g$-PEA beads prepared in acidic TPP solution can be dominated completely by ionic-crosslinking $[35,36]$. Based on this fact, in this work, CMC-gPEA beads were prepared with TPP at $\mathrm{pH} 4$. The surface morphologies of control and drug loaded CMC- $g$-PEA beads are shown in Fig. 4. The control CMC- $g$-PEA beads were about $800-1000 \mu \mathrm{m}$ in size and roughly spherical in shape, and appeared to have smooth outer surfaces (Fig. 4a and b). These beads were dense when compared to the beads loaded with drug due to the higher crosslinking density. Incorporation of drug into the beads altered the surface morphology of CMC-g-PEA beads. The results revealed that addition of drug imparts a high degree of surface roughness to the beads (Figs. 4c-f). With regard to the beads size, it notably varied depending on the feed ratio: as the feed ratio increased, the bead size increased from 1000 to $1200 \mu \mathrm{m}$. Table 1 shows the effect of feed ratio on loading efficiency. The feed ratio of ketoprofen to CMC- $g$-PEA ranged from 0.5 to 1 . The results demonstrated that the loading efficiency defined as the percentage of entrapped ketoprofen to $\mathrm{CMC}-g$-PEA is in the range of $68-82 \%$ and the increase in feed ratio improves the loading content.

\subsection{Swelling behaviour}

The swelling behaviour of the chitosan and CMC- $g$-PEA beads in acidic ( $\mathrm{pH} 1.4)$ and basic $\left(\mathrm{pH} \mathrm{7.4)}\right.$ solutions at $37^{\circ} \mathrm{C}$ is shown in Fig. 5. It is evident that the character of swelling curves for both chitosan and CMC-g-PEA beads changes significantly over time in $\mathrm{pH} 1.4$ compared to that in $\mathrm{pH}$ 7.4. Moreover, the swelling can reach a stable equilibrium more rapidly in $\mathrm{pH} 7.4$ than in $\mathrm{pH}$ 1.4. The swelling behaviour of chitosan beads at pH 1.4 was found to be preserved within $24 \mathrm{~h}$, followed by dissolution after $48 \mathrm{~h}$. Generally, the swelling process of the chitosan beads at a low $\mathrm{pH}$ involves protonation of amino groups in the beads $[37,38]$. Therefore, the repulsive force between the positive charges of protonated amine groups of chitosan molecules resulted in longer intermolecular distances which would be responsible for higher swelling degree and rapid disintegration of chitosan particles. On the other hand, the observed swelling rate of CMC-g-PEA beads is found to be slower than that of control chitosan beads and also CMC- $g$-PEA beads were found to maintain their shape for $72 \mathrm{~h}$ and only after $72 \mathrm{~h}$ most of the beads were dissolved in the medium. The slow swelling 

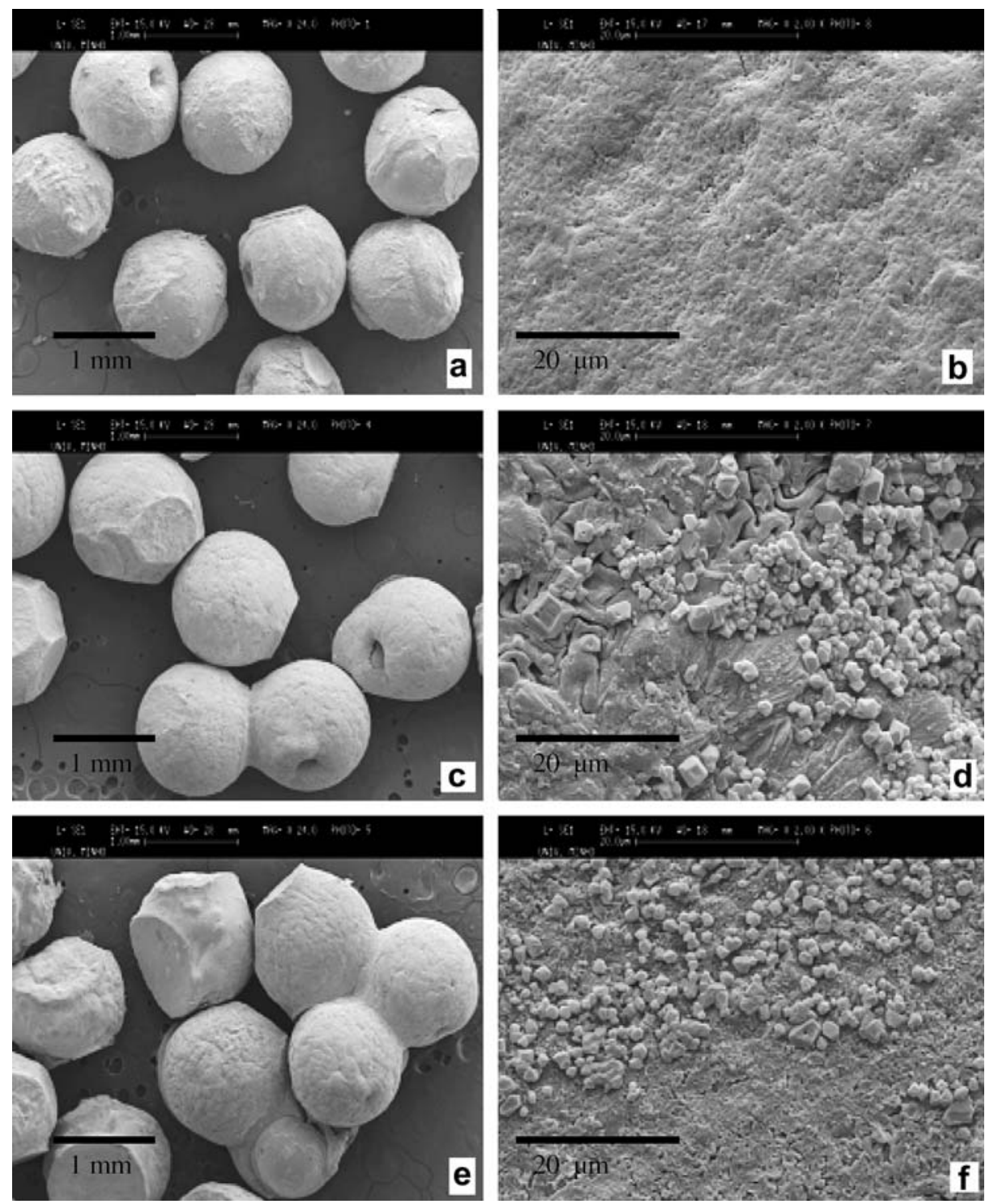

Fig. 4. SEM micrographs of (a) CMC- $g$-PEA beads and (b) its surface morphology, (c) beads loaded with drug (1:0.5 ratio) and (d) its surface morphology, (e) beads loaded with drug (1:1) ratio and (f) its surface morphology.

Table 1

Effect of feed ratio on loading efficiency

\begin{tabular}{llllr}
\hline $\begin{array}{l}\text { CMC-g-PEA } \\
(\mathrm{mg} / \mathrm{ml})\end{array}$ & $\begin{array}{l}\text { Ketoprofen } \\
(\mathrm{mg} / \mathrm{ml})\end{array}$ & $\begin{array}{l}\text { Loading } \\
\text { efficiency }^{\mathrm{a}} \\
(\%)\end{array}$ & $\begin{array}{l}\text { Loading } \\
\text { content } \\
(\%)\end{array}$ & $\begin{array}{l}\text { Size }^{\mathrm{c}} \\
(\mu \mathrm{m})\end{array}$ \\
\hline 10 & - & - & - & 900 \\
10 & 5 & 68 & 29 & 1100 \\
10 & 10 & 82 & 69 & 1200 \\
\hline
\end{tabular}

${ }^{\text {a }}$ Percentage of loaded ketoprofen, based on feed amount.

b Weight percentage of ketoprofen to CMC-g-PEA.

${ }^{\mathrm{c}}$ Mean diameter. rate and high stability of CMC- $g$-PEA beads at $\mathrm{pH}$ 1.4 may be due to the complexity involved through the inter/intra molecular bondings between the grafted PEA side chains. The successful oral drug carrier must be resistant to the impact of the $\mathrm{pH}$ gradients due to $\mathrm{pH}$ changing from the strongly acidic medium in the stomach to the weekly alkaline medium in the intestine. Fig. 5a clearly showed that CMC-g-PEA beads have higher stability than chitosan beads at pH 1.4 and may be suitable for oral and control drug delivery applications. The swelling 

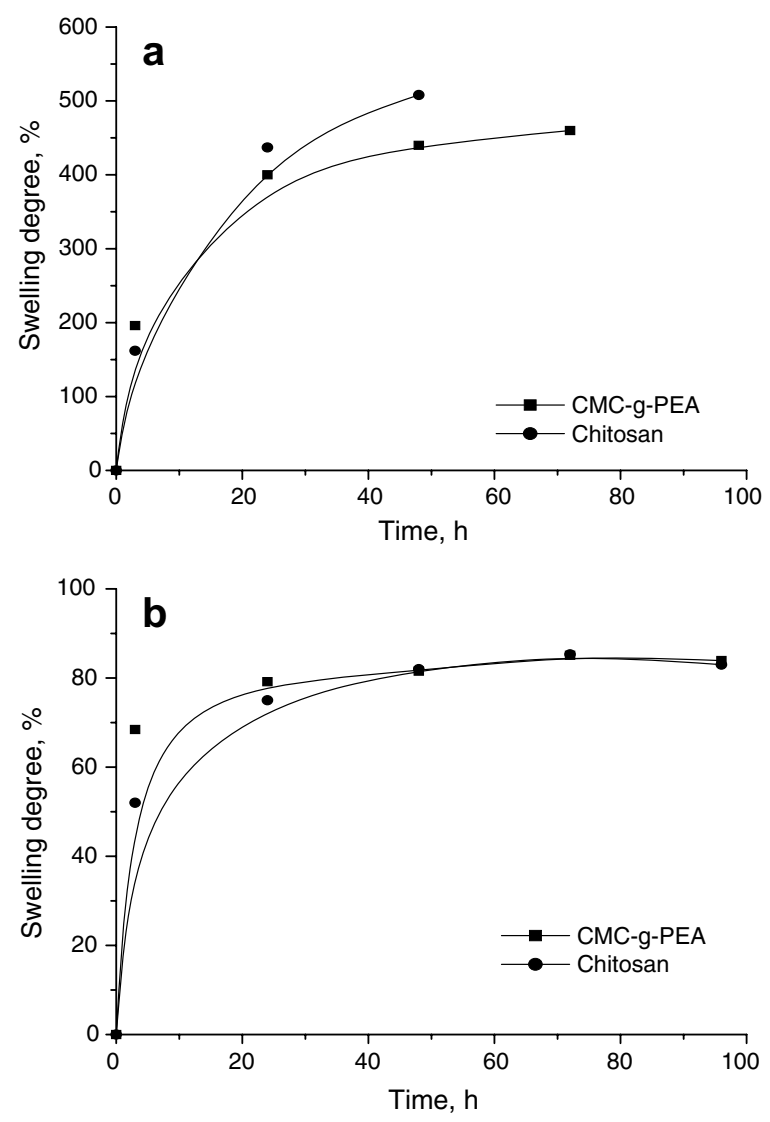

Fig. 5. Swelling behaviour of CMC-g-PEA and Chitosan beads at (a) $\mathrm{pH} 1.4$ and (b) 7.4.

capability of CMC- $g$-PEA beads was found to be similar to that of chitosan at pH 7.4 (Fig. 5b). However, CMC-g-PEA beads reached a stable equilibrium more rapidly (within $24 \mathrm{~h}$ ) than chitosan (within $48 \mathrm{~h}$ ). The swelling degree of both chitosan and CMC-g-PEA beads tends to decline after the beads were swollen for some time, which may indicate that the dissolution tendency of the beads exceeds the swelling degree [39].

\subsection{Drug release behaviour}

The release of ketoprofen from CMC-g-PEA and chitosan beads was carried out in acidic $(\mathrm{pH} 1.4)$ and basic ( $\mathrm{pH} 7.4$ ) solutions at $37^{\circ} \mathrm{C}$. Figs. 6 shows the release profiles of ketoprofen from CMC- $g$-PEA and chitosan beads loaded with $29 \%$ and $69 \%$ of drug as a function of time. The control chitosan beads, which have less interaction with the drug molecules, showed a faster release $\left(t_{80 \%} \sim 7-12 \mathrm{~h}\right)$ and rapidly disintegrated, whereas those based on
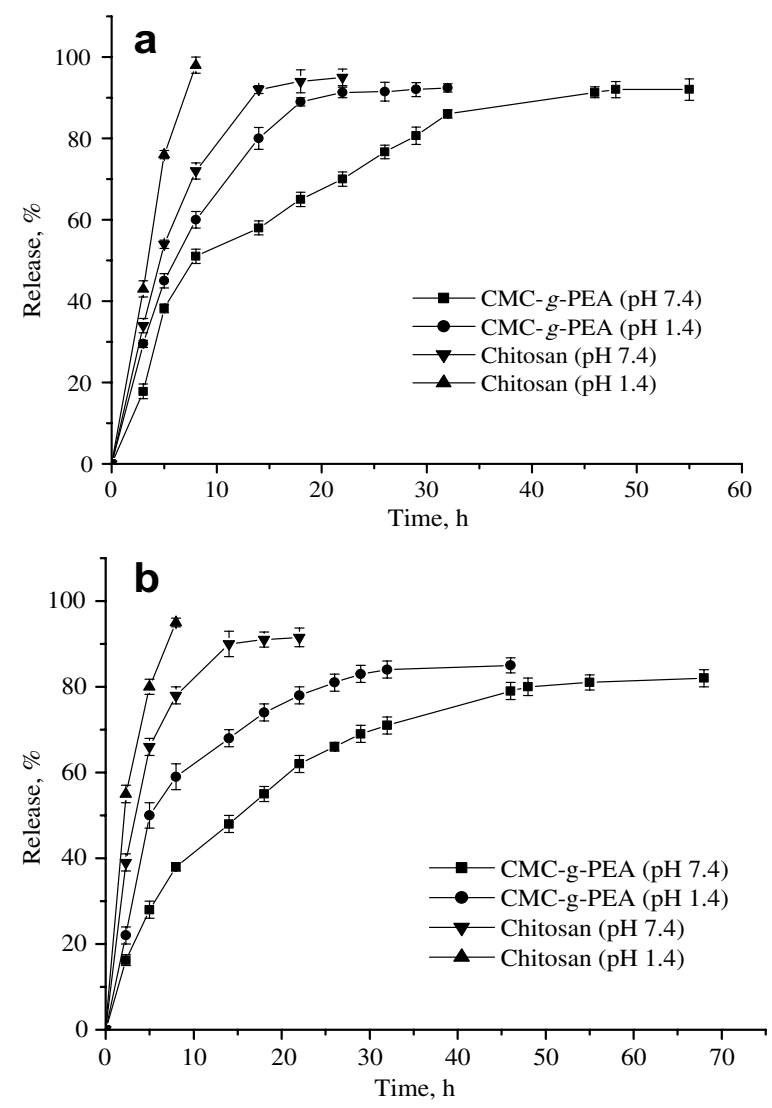

Fig. 6. Release of ketoprofen from (a) $29 \%$ and (b) $69 \%$ drug loaded chitosan and CMC- $g$-PEA beads.

CMC- $g$-PEA remained intact in aqueous medium and showed a slow and steady release $\left(t_{80 \%} \sim 15-\right.$ $30 \mathrm{~h}$ ), being the equilibrium reached after $20 \mathrm{~h}$. In the CMC- $g$-PEA beads, the water-insoluble nature of ketoprofen, ascribed to their hydrophobicity, might govern the release pattern, accompanied with physical interaction with CMC-g-PEA such as hydrophobic interaction, hydrogen bonding, and electrostatic interaction. In an aqueous solution, micelles or self-aggregates can be formed by the amphiphilic nature of the CMC- $g$-PEA due to their intra and inter molecular hydrophobic interactions [40]. The strong interaction of the hydrophobic cores of micelles or self-aggregates with the hydrophobic drug molecules could be a responsible for the slow release of drug from CMC- $g$-PEA beads. Here, the hydrophobic core serves as a reservoir for hydrophobic drug molecules, whereas the hydrophilic shell affects their pharmacokinetic behaviour. In addition, the slow release may also be attributed to a hydrophobic barrier limiting 
access of water and dissolution of the drug. The amount and percentage of drug released from both the CMC- $g$-PEA and chitosan beads was $\mathrm{pH}$-dependent and occurs at higher rate in acidic medium than in basic medium. This can be explained considering the rate of diffusion from the swollen beads in acidic and basic media. In basic medium ( $\mathrm{pH} 7.4)$, there is a limited swelling of the beads which inhibits the diffusion of drugs at a faster rate as it occurs in acidic medium ( $\mathrm{pH}$ 1.4). Initially, the magnitude of swelling of beads in acidic medium is very high and gives rise to a significant burst effect through uncontrolled diffusion, but becomes almost constant due to controlled diffusion at beads equilibrium swelling. In the acidic medium, the rate of drug release from chitosan beads was found to be higher than that of CMC-g-PEA beads. A higher swelling of the chitosan beads due to their longer intermolecular distances caused by the repulsive force between the protonated amine groups at the acidic medium enhances the diffusion of the drugs at a faster rate. But in the case of CMC-g-PEA beads, the drug release would be decreased due to the hydrophobic interaction of drug with CMC-g-PEA as mentioned before.

The release profile of the highly drug loaded beads (Fig. 6b) has been found to be similar to that of the beads, loaded with lower amounts of the drug (Fig. 6a). In both cases, an initial burst release followed by a slow release of ketoprofen occurred. The results have shown that the percentage of drug released from CMC-g-PEA beads has decreased with the increase in concentration of ketoprofen. However, as expected, the total amount of drug released was found to be higher from the highly loaded beads in comparison to the beads loaded with low concentrations. The kinetics of drug release by CMC- $g$-PEA beads was dependent on the initial drug loading. For beads with $29 \%$ of drug, the duration of release was up to $45 \mathrm{~h}$. For $69 \%$ drug loads, the duration of release increased to $50 \mathrm{~h}$. The explanation of this phenomenon may reside with the drug solubility, particularly in the case of hydrophobic drugs that alter the hydrophobic/hydrophilic character of the system [23]. At high drug loading $(69 \%)$, there is a reduced level of hydration that would give rise to a slow release of the drug.

According to Peppas $[41,42]$, there are three primary mechanisms by which the release of active agents can be controlled: erosion, diffusion, and swelling followed by diffusion. Erosion may take place via hydration or hydrolysis of the bulk, the polymer being slowly degraded starting at the periphery of the particle. Diffusion can occur through the unhydrated polymer matrix but will generally be facilitated as the polymer gradually swells in contact with the body fluids. In the case of CMC- $g$-PEA beads, it was observed that drug release is quite fast at the initial period of time. About $40-60 \%$ of the drug is released in about $10 \mathrm{~h}$. Then, almost a constant and much slower release rate is observed. Notice that the swelling curves are quite similar to the release curves. It seems that release obeys a swelling-controlled release mechanism, especially at this initial period of release. After this initial period, in which the swelling equilibrium is achieved, the release is most probably followed by a diffusion-controlled mechanism. This is an expected behaviour found in the case of hydrogels during drug release studies; first a hydration followed by polymer chain relaxation takes place by water penetration into the matrix. During this process, high release rates occur due to the presence of the drug at and near the surface. After forming the gel structure the release continues with the diffusion mechanism through the matrix by a much slower release rate. This typical phenomenon is the same in the case of drug release from CMC-g-PEA beads. However, there is another controlling parameter for the drug release profile, conferred by the ionic nature of the crosslinking present in between the polymer chains. The disruption of such linkage may accelerate the release kinetic especially in the later stages that may help for maintaining a more stable delivery of the drug with time. Note that this process could be controlled by changing the concentration of crosslinking agent.

\section{Conclusions}

Chemically modified chitosans have potential to be used in controlled drug delivery system. In this work, CMC was hydrophobically modified by grafting with PEA in order to produce a system containing amphiphilic capability. Due to this character, CMC-g-PEA can be used as matrices for the controlled delivery of hydrophobic drugs. The grafting reaction was confirmed by FT-IR spectroscopy and ${ }^{1} \mathrm{H}$ NMR. For the release study, the CMC-g-PEA beads were prepared by an ionic gelation method. The CMC-g-PEA beads show a pH-dependent swelling behaviour, which makes them appropriate 
for $\mathrm{pH}$-responsive delivery of drugs. The preliminary results for ketoprofen as a model drug, including loading and release experiments, indicate that this system seems to be a very promising vehicle for the administration of controlled release of hydrophobic drugs with a $\mathrm{pH}$-responsive capability. The strong hydrophobic interaction of CMC- $g$-PEA with the drug molecules could be a main reason for the slow and steady release of the drug from CMC$g$-PEA beads. It is believed that the release of drug may be controlled by swelling followed by diffusioncontrolled mechanism.

\section{Acknowledgements}

M. Prabaharan thanks the Portuguese Foundation for Science and Technology (FCT), for awarding a post-doctoral research grant (SFRH/BPD/ 14671/2003). Financial support for this work was provided by FCT, through the POCTI and FEDER programmes and by the European Union funded STREP Project HIPPOCRATES (NMP3-CT2003-505758).

\section{References}

[1] H.S. Blair, T.C. Ho, J. Chem. Tech. Biotechnol. 31 (1980) 6.

[2] M. Prabaharan, J.P. Borges, M.H. Godinho, J.F. Mano, Mater. Sci. Forum 514-516 (2006) 1010.

[3] D.C. Hwang, S. Damodaran, J. Agric. Food Chem. 43 (1995) 33.

[4] Y. Shigemasa, K. Saito, H. Sashiwa, H. Saimoto, Int. J. Biol. Macromol. 16 (1994) 43.

[5] J.K. Suh, H.W. Matthew, Biomaterials 21 (2000) 2589.

[6] S.C. Bhatia, N.A. Ravi, Biomacromolecules 1 (2000) 413.

[7] M.T. Chiang, H.T. Yao, H.C. Chen, Biosci. Biotechnol. Biochem. 64 (2000) 965.

[8] N. Koseva, N. Manolova, N. Markova, T. Radoucheva, I. Rashkov, Polym. Bull. 43 (1999) 101.

[9] M. Prabaharan, J.F. Mano, Drug Delivery 12 (2005) 41.

[10] R. Jayakumar, M. Prabaharan, R.L. Reis, J.F. Mano, Carbohydr. Polym. 62 (2005) 142.

[11] M. Prabaharan, J.F. Mano, Carbohydr. Polym. 63 (2006) 153.

[12] F.L. Mi, S.S. Shyu, C.T. Chen, J.Y. Lai, Polymer 43 (2002) 757.

[13] S. Tokura, H. Tamura, Biomacromolecules 2 (2001) 417.
[14] W. Xie, P. Xu, Q. Liu, Bioorg. Med. Chem. Lett. 11 (2001) 1699.

[15] H. Sashiwa, J.M. Thompson, S.K. Das, Y. Shigemasa, S. Tripathy, R. Roy, Biomacromolecules 1 (2000) 303.

[16] B.O. Jung, C.H. Kim, K.S. Choi, J.J. Kim, J. Appl. Polym. Sci. 72 (1999) 1713.

[17] S. Hirano, Y. Tanaka, M. Hasegawa, K. Tobetto, A. Nishioka, Carbohyd. Res. 29 (1985) 205.

[18] J.A. Hubbell, Science 300 (2003) 595.

[19] C.G. Liu, X.G. Chen, H.J. Park, Carbohyd. Polym. 62 (2005) 293.

[20] K.Y. Lee, I.C. Kwon, Y.H. Kim, W.H. Jo, S.Y. Jeong, J. Control. Release 51 (1998) 213.

[21] J.H. Park, S. Kwon, J.O. Nam, R.W. Park, H. Chung, S.B. Seo, I.S. Kim, I.C. Kwon, S.Y. Jeong, J. Control. Release 95 (2004) 579.

[22] J.H. Park, S. Kwon, M. Lee, H. Chung, J.H. Kim, Y.S. Kim, R.W. Park, I.S. Kim, S.B. Seo, I.C. Kwon, S.Y. Jeong, Biomaterials 27 (2006) 119.

[23] C.L. Tien, M. Lacroix, P.I. Szabo, M.A. Mateescu, J. Control. Release 93 (2003) 1.

[24] L. Noble, A.I. Gray, L. Sadiq, I.F. Uchegbu, Int. J. Pharm. 192 (1999) 173.

[25] L. Martin, C.G. Wilson, F. Koosha, I.F. Uchegbu, Eur. J. Pharm. Biopharm. 55 (2003) 35.

[26] M. Prabaharan, J.F. Mano, Macromol. Biosci. 5 (2005) 965.

[27] S.A. Agnihotri, N.N. Mallikarjuna, T.M. Aminabhavi, J. Control. Release 100 (2004) 5.

[28] L. Chen, Z. Tian, Y. Du, Biomaterials 25 (2004) 3725.

[29] I. Genta, P. Perugini, B. Conti, F. Pabanetto, Int. J. Pharm. 152 (1997) 183.

[30] F.L. Mi, S.S. Shyu, C.T. Chen, J.Y. Schoung, Biomaterials 20 (1999) 1603.

[31] X.Z. Shu, K.J. Zhu, J. Microencapsulation 18 (2001) 237.

[32] X.Z. Shu, K.J. Zhu, Int. J. Pharm. 201 (2000) 51.

[33] P. Calvo, R.C. Lopez, V.J.L. Jato, M.J. Alonso, J. Appl. Polym. Sci. 63 (1997) 125.

[34] F.L. Mi, S.S. Shyu, C.Y. Kuan, S.T. Lee, K.T. Lu, S.F. Jang, J. Appl. Polym. Sci. 74 (1999) 1868.

[35] F.L. Mi, S.S. Shyu, S.T. Lee, T.B. Wong, J. Polym. Sci: Polym. Phys. 37 (1999) 1551.

[36] S.T. Lee, F.L. Mi, Y.J. Shen, S.S. Shyu, Polymer 42 (2001) 1879.

[37] K.C. Gupta, M.N.V. Ravi Kumar, Polym. Int. 49 (2000) 41.

[38] K.C. Gupta, M.N.V. Ravi Kumar, Biomaterials 21 (2000) 1115.

[39] K.C. Gupta, M.N.V. Ravi Kumar, JMS-Pure Appl. Chem. 36A (1999) 827.

[40] G.M. Whitesides, J.P. Mathias, C.T. Sato, Science 254 (1991) 1312.

[41] N.A. Peppas, Pharm. Acta Helv. 60 (1985) 110

[42] L.B. Peppas, Med. Plast. Biomater. 4 (1997) 34 